Supporting Information

\title{
A Platinum Acetylide Polymer with Sterically Demanding Substituents: Effect of Aggregation on the Triplet Excited State
}

\author{
Xiaoming Zhao, Thomas Cardolaccia, Richard Farley, \\ Khalil A. Abboud and Kirk S. Schanze* \\ Department of Chemistry, University of Florida \\ PO Box 117200, Gainesville, FL 32601-7200
}

* Author to whom correspondence should be addressed

TEL: 352-392-9133

FAX: 352-392-2395

e-mail: kschanze@chem.ufl.edu 


\section{X-Ray Structure of 1a}

Data were collected at $173 \mathrm{~K}$ on a Siemens SMART PLATFORM equipped with A CCD area detector and a graphite monochromator utilizing $\operatorname{MoK}_{\alpha}$ radiation $(\lambda=0.71073 \AA)$. Cell parameters were refined using up to 8192 reflections. A full sphere of data (1850 frames) was collected using the $\omega$-scan method $\left(0.3^{\circ}\right.$ frame width). The first 50 frames were remeasured at the end of data collection to monitor instrument and crystal stability (maximum correction on I was $<1 \%)$. Absorption corrections by integration were applied based on measured indexed crystal faces.

The structure was solved by the Direct Methods in SHELXTL6, ${ }^{1}$ and refined using fullmatrix least squares. The non- $\mathrm{H}$ atoms were treated anisotropically, whereas the hydrogen atoms were calculated in ideal positions and were riding on their respective carbon atoms. The asymmetric unit consists of a complex in general position and two half complexes around centers of inversion. A total of 1827 parameters were refined in the final cycle of refinement using 80376 reflections with $\mathrm{I}>2 \sigma(\mathrm{I})$ to yield $\mathrm{R}_{1}$ and $\mathrm{wR}_{2}$ of $5.80 \%$ and $10.78 \%$, respectively. Refinement was done using $\mathrm{F}^{2}$.

(1) Sheldrick, G. M. (2000). SHELXTL6. Bruker-AXS, Madison, Wisconsin, USA 
Table S-1. Crystal data and structure refinement for $1 \mathrm{a}$.

Identification code

Empirical formula

Formula weight

Temperature

Wavelength

Crystal system

Space group

Unit cell dimensions

Volume

Z

Density (calculated)

Absorption coefficient

$\mathrm{F}(000)$

Crystal size

Theta range for data collection

Index ranges

Reflections collected

Independent reflections

Completeness to theta $=27.50^{\circ}$

Absorption correction

Max. and min. transmission

Refinement method

Data / restraints / parameters

Goodness-of-fit on $\mathrm{F}^{2}$

Final $\mathrm{R}$ indices [I $>2 \operatorname{sigma}(\mathrm{I})]$

$\mathrm{R}$ indices (all data)

Largest diff. peak and hole $\mathrm{xz} 01$

C272 H400 Cl6 P12 Pt6

5424.80

173(2) K

$0.71073 \AA$

Triclinic

P-1

$\mathrm{a}=11.9307(8) \AA \quad \alpha=72.186(2)^{\circ}$.

$\mathrm{b}=24.2701(15) \AA \quad \beta=89.427(2)^{\circ}$.

$\mathrm{c}=32.747(2) \AA \quad \gamma=85.613(2)^{\circ}$.

$9000.1(10) \AA^{3}$

2

$2.002 \mathrm{Mg} / \mathrm{m}^{3}$

$4.915 \mathrm{~mm}^{-1}$

5564

$0.342 \times 0.171 \times 0.114 \mathrm{~mm}^{3}$

0.88 to $27.50^{\circ}$.

$-15 \leq \mathrm{h} \leq 15,-31 \leq \mathrm{k} \leq 31,-42 \leq \mathrm{l} \leq 41$

80376

$40118[\mathrm{R}(\mathrm{int})=0.0749]$

$97.0 \%$

Integration

0.6031 and 0.2294

Full-matrix least-squares on $\mathrm{F}^{2}$ 40118 / 0 / 1827

1.152

$\mathrm{R} 1=0.0580, \mathrm{wR} 2=0.1078$ [31761]

$\mathrm{R} 1=0.0794, \mathrm{wR} 2=0.1150$

1.972 and -1.534 e. $\AA^{-3}$

$$
\begin{aligned}
& \mathrm{R} 1=\sum\left(\left\|\mathrm{F}_{\mathrm{O}}|-| \mathrm{F}_{\mathrm{C}}\right\|\right) / \sum\left|\mathrm{F}_{\mathrm{O}}\right| \\
& \mathrm{wR} 2=\left[\sum\left[\mathrm{w}\left(\mathrm{F}_{\mathrm{O}}^{2}-\mathrm{Fc}^{2}\right)^{2}\right] / \sum\left[\mathrm{w}\left(\mathrm{F}_{\mathrm{O}}^{2}\right)^{2}\right]\right]^{1 / 2} \\
& \mathrm{~S}=\left[\sum\left[\mathrm{w}\left(\mathrm{F}_{\mathrm{O}}^{2}-\mathrm{F}_{\mathrm{c}}^{2}\right)^{2}\right] /(\mathrm{n}-\mathrm{p})\right]^{1 / 2} \\
& \mathrm{w}=1 /\left[\sigma^{2}\left(\mathrm{~F}_{\mathrm{O}}^{2}\right)+\left(\mathrm{m}^{*} \mathrm{p}\right)^{2}+\mathrm{n}^{*} \mathrm{p}\right], \mathrm{p}=\left[\max \left(\mathrm{F}_{\mathrm{O}}^{2}, 0\right)+2^{*} \mathrm{~F}_{\mathrm{c}}^{2}\right] / 3, \mathrm{~m} \& \mathrm{n} \text { are constants. }
\end{aligned}
$$




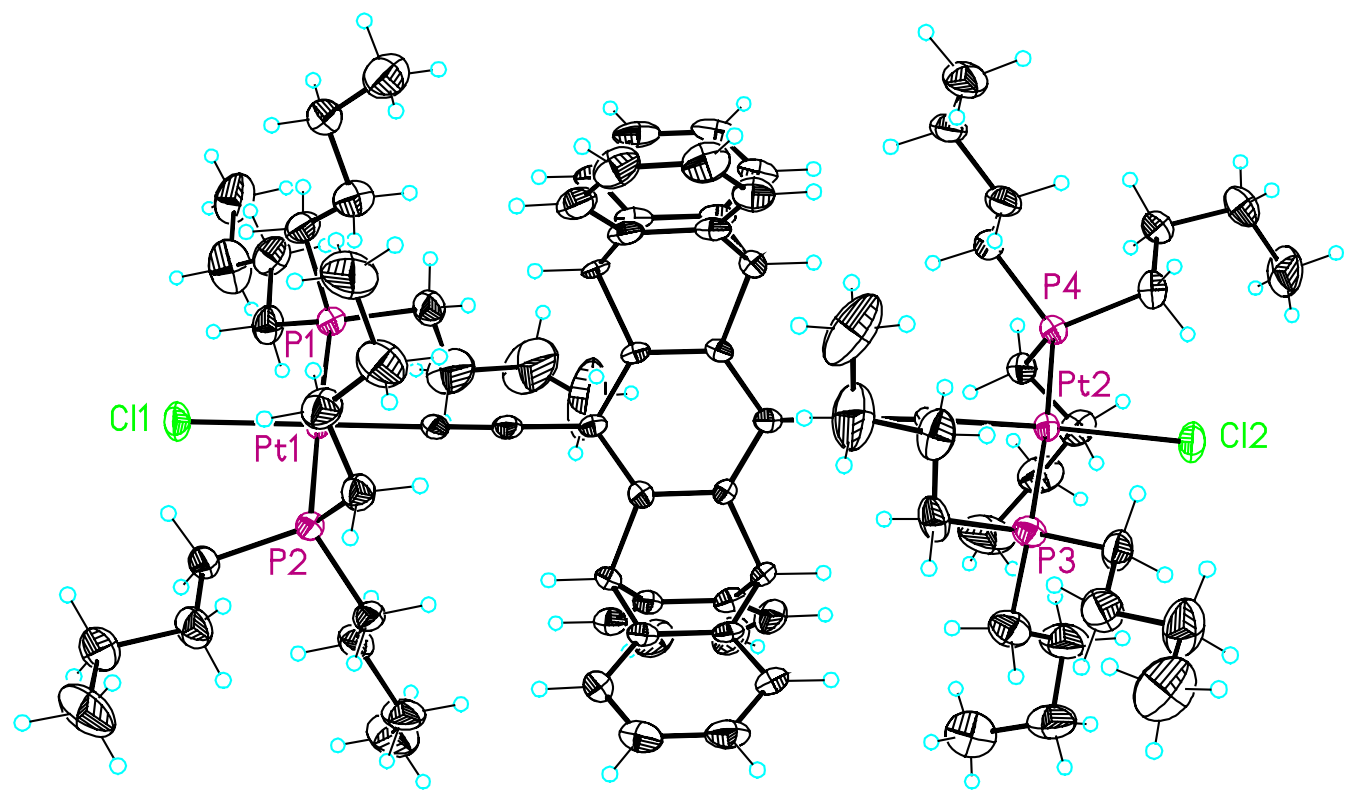

Figure S-1. ORTEP diagram of compound 1a. 

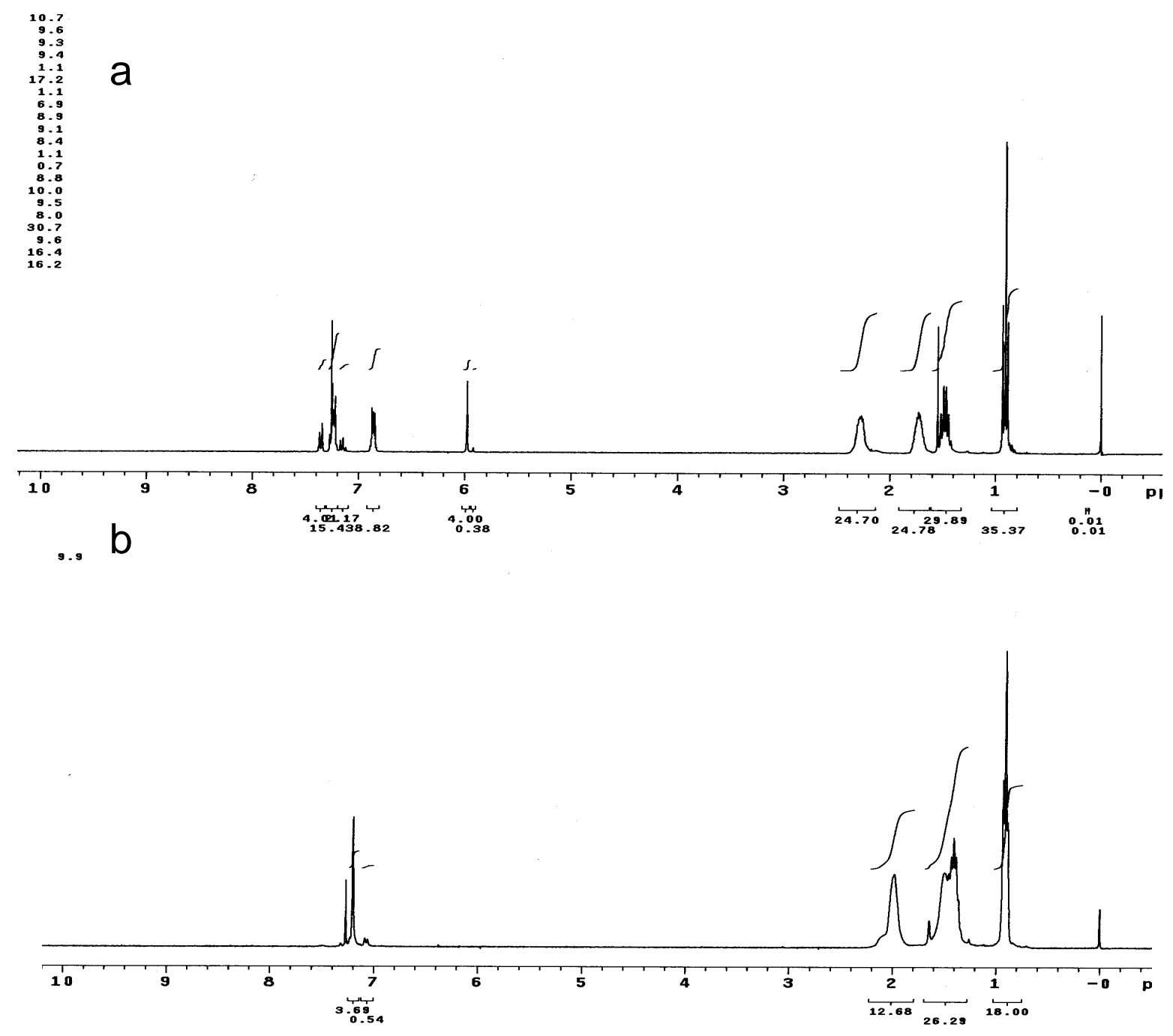

C

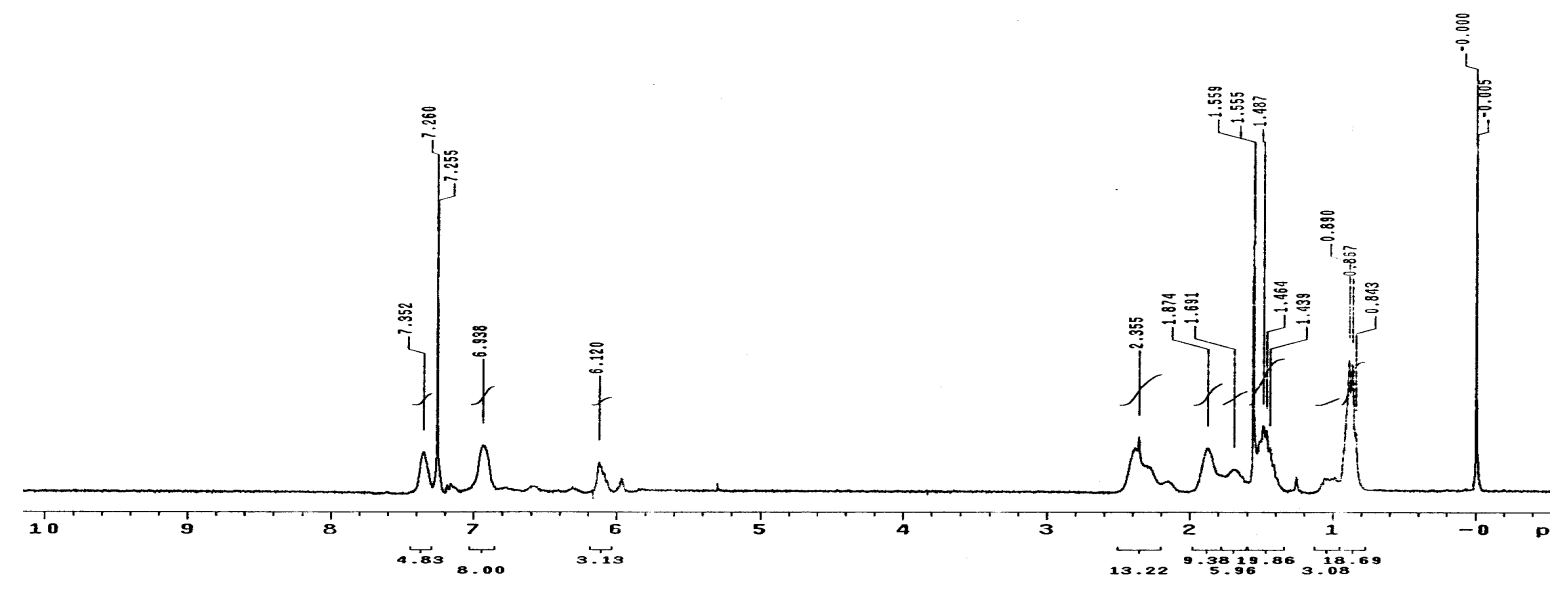

Figure S-2. ${ }^{1} \mathrm{H}$ NMR spectra obtained in $\mathrm{CDCl}_{3}$ solution at $300 \mathrm{MHz}$. (a) Monomer 1b. (b) Polymer 2. (c) Polymer 3. 


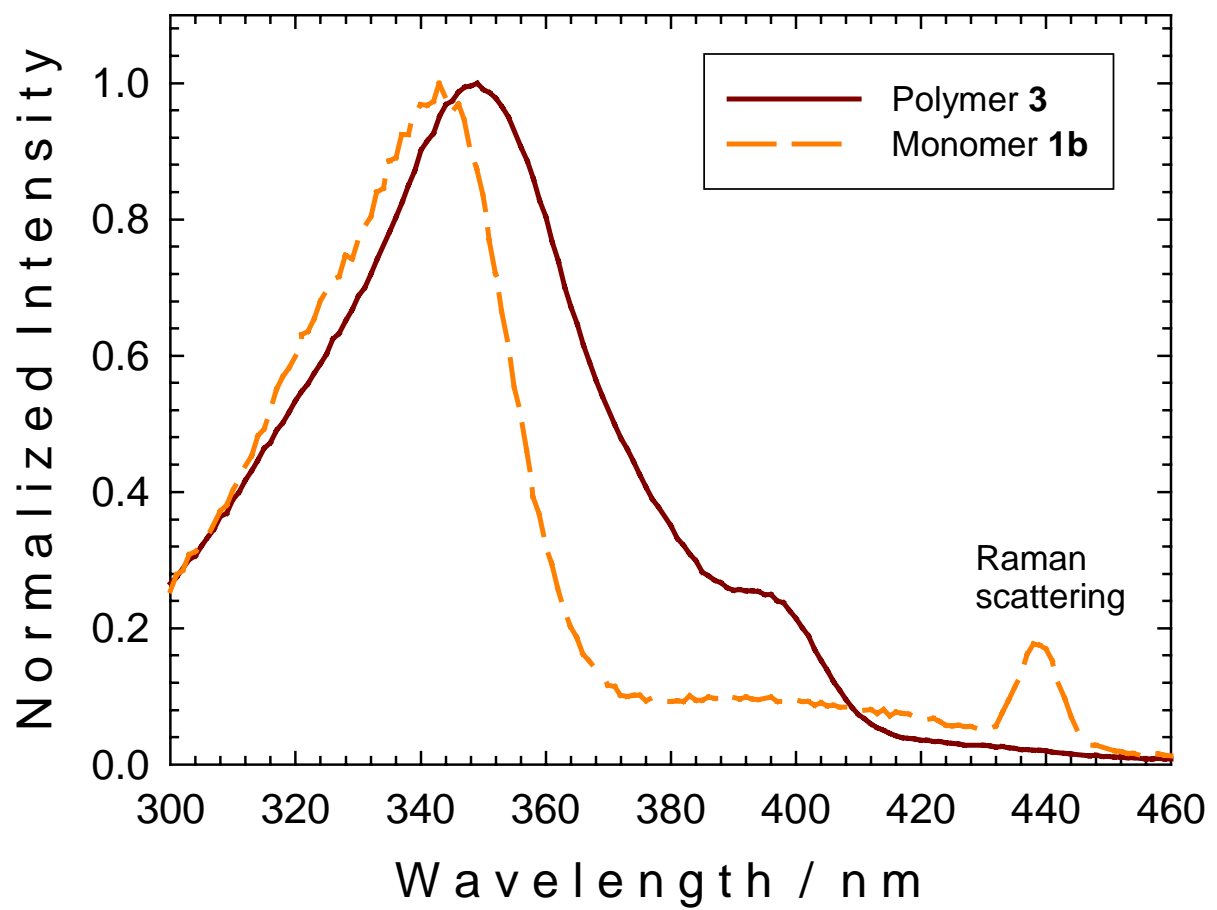

Figure S-3. Emission excitation spectra obtained for THF solutions of $\mathbf{1 b}$ and $\mathbf{3}$. Excitation scan obtained while monitoring emission maximum. $\left(\mathbf{1 b}: \boldsymbol{\lambda}_{\mathrm{em}}=490 \mathrm{~nm}, \mathbf{3}\right.$ : $\lambda_{\mathrm{em}}=562 \mathrm{~nm}$.) 


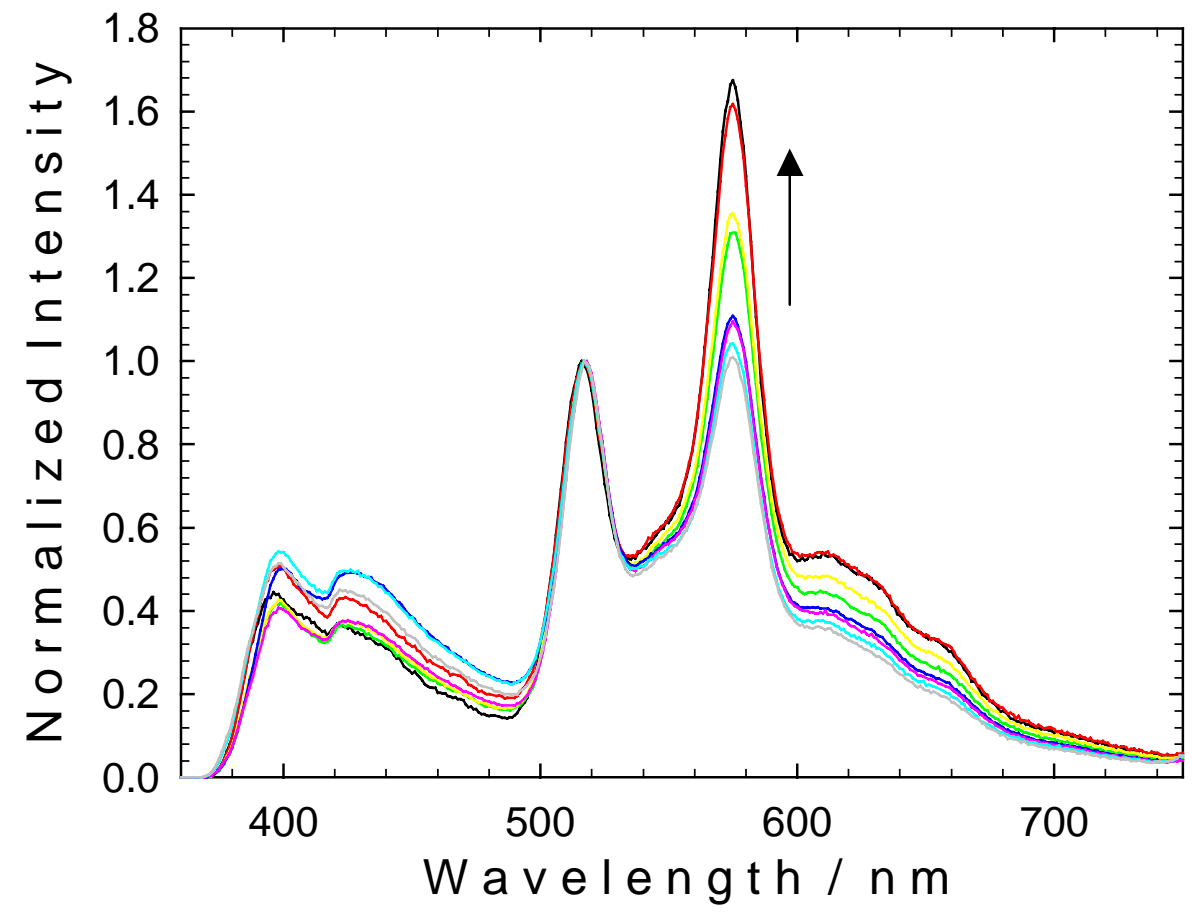

Figure S-4. Emission spectra of polymer $\mathbf{2}$ in THF solution at various concentrations, excitation wavelength $333 \mathrm{~nm}$. Arrow indicates change of spectrum with increasing concentration. Concentration range : $0.1-1.0 \mu \mathrm{M}$ (polymer repeat unit). Spectra normalized to intensity at $515 \mathrm{~nm}$ to facilitate comparison. 